\title{
Efeito do exercício resistido na redução do edema, nocicepção e regeneração nervosa em um modelo de compressão do nervo isquiático
}

\author{
Effect of resistance training on the reduction of edema, nociception, \\ and nerve regeneration in a sciatic nerve compression model
}

Juliana Sobral Antunes', Keli Lovison' ${ }^{1}$, Jhenifer Karvat ${ }^{1}$, Lisyana Vieira ${ }^{1}$, Ana Luiza Peretti ${ }^{1}$, Guilherme Hideaki Higuchi ${ }^{1}$, Maria Lúcia Bonfleur ${ }^{1}$, Rose Meire Costa Brancalhão', Lucinéia de Fátima Chasko Ribeiro', Gladson Ricardo Flor Bertolini ${ }^{1} \bowtie$

${ }^{1}$ Universidade Estadual do Oeste do Paraná (Unioeste). Cascavel, PR.

\section{RESUMO}

Objetivos: Analisar os efeitos do exercício físico resistido de subida em escada, sobre o edema, nocicepção e regeneração nervosa de ratos Wistar, submetidos à compressão do nervo isquiático.

Métodos: Foram estudados 24 ratos Wistar, divididos igualmente entre quatro grupos: Grupo Controle, Grupo Exercício, Grupo Lesão e Grupo Tratado - Lesão e Exercício. O Grupo Lesão e o Grupo Tratado foram submetidos à compressão do nervo isquiático com pinça hemostática por 30 segundos. A partir do terceiro dia após a lesão, iniciou-se o tratamento com exercício resistido de subida em escada para o Grupo Exercício e o Grupo Tratado. O tratamento consistiu em realizar duas séries de 10 subidas na escada, com sobrecarga de 100 gramas e intervalo de um minuto entre uma série e outra. $\mathrm{O}$ estudo foi conduzido por 22 dias e nesse tempo os animais foram avaliados quanto ao edema e à nocicepção. No $22^{\mathrm{O}}$ dia de pós-operatório, os animais foram anestesiados para retirada de um fragmento do nervo isquiático para análise do número de axônios e da densidade de fibras. Em seguida, ainda sob efeito da anestesia, os animais foram eutanasiados. Os nervos coletados seguiram protocolo de processamento histológico de rotina. As expressões do Fator de Crescimento Neural e do Fator de Crescimento Derivado do Cérebro foram avaliadas por Western blotting.

Resultados: Não houve diferença significativa entre os grupos no tamanho do edema. O Grupo Controle apresentou maior limiar nociceptivo comparado aos demais grupos. A análise morfométrica não revelou diferença significativa entre os grupos, quanto à quantidade de axônios e à densidade de fibras. A expressão do Fator de Crescimento Derivado do Cérebro foi maior no Grupo Lesão e no Grupo Tratado quando comparados ao Grupo Controle.

Conclusões: O exercício físico resistido de subida em escada, nos parâmetros propostos, não foi eficaz para reduzir o edema, a nocicepção ou aumentar o número de axônios e a densidade de fibras nervosas após lesão do nervo isquiático.

DESCRITORES: atividade motora; nervo isquiático; neuropatia ciática; medição da dor; regeneração nervosa.

\section{ABSTRACT}

Aims: To analyze the effects of ladder-climbing resistance training exercise on edema, nociception, and regeneration of the sciatic nerve in Wistar rats subjected to sciatic nerve compression.

Methods: Twenty-four Wistar rats were divided into four groups: Control Group, Exercise Group, Injury Group, and Treated Group (injury and exercise). Injury Group and Treated Group were subjected to sciatic nerve compression with a hemostat for 30 seconds. On the third day after injury, Exercise Group and Treated Group began treatment with ladder-climbing resistance exercise. The treatment consisted in performing two series of 10 ladder climbs with a 100-gram overload and a one-minute interval between the series. The study was conducted for 22 days, during which time the animals were evaluated for edema and nociception. Twenty-two days after surgery, the animals were anesthetized for removal of a sciatic nerve fragment and analysis of the number of axons and fiber density. Thereafter, still under anesthesia, the animals were euthanized. Nerve sampling followed the routine histological processing protocol. Expressions of Neural Growth Factor and Brain-derived Neurotrophic Factor were evaluated by Western blotting.

Results: There was no significant difference in edema size between groups. Control Group showed the highest nociceptive threshold compared to the other groups. The morphometric analysis showed no significant difference in number of axons and fiber density between groups. The expression of Brain-derived Neurotrophic Factor was greater in the Injury Group and the Treated Group compared to the Control Group.

Conclusions: The proposed ladder-climbing resistance training was not effective in reducing edema and nociception or in increasing the number of axons and fiber density after sciatic nerve injury.

KEY WORDS: motor activity; sciatic nerve; sciatic neuropathy; pain measurement; nerve regeneration. 
Abreviaturas: GC, grupo controle; GE, grupo exercício; GL, grupo lesão; GLE, grupo tratado (lesão e exercício); PO, pós-operatório; AVE, avaliação do edema; AVN, avaliação da nocicepção; Q, quadrante; IL, Interleucina; NGF, fator de crescimento neural; BDNF, fator de crescimento dervivado do cérebro.

\section{INTRODUÇÃO}

A dor lombar é uma disfunção prevalente em aproximadamente $11,9 \%$ da população mundial, sendo que em alguns casos, pode ocorrer o comprometimento do nervo isquiático gerando dor ao longo do trajeto do mesmo, o que caracteriza um quadro de ciatalgia. As causas gerais da ciatalgia são hérnias de disco, infecções, compressão nervosa, anomalias congênitas, doenças neurológicas degenerativas e disfunções musculoesqueléticas, entre outras. Essa condição pode resultar em deficiências físicas e diminuição da qualidade de vida e da capacidade produtiva do indivíduo [1-3].

Os tratamentos para lesões que atingem o sistema nervoso periférico podem ser clínicos ou cirúrgicos. Ultimamente tem sido dada atenção especial a abordagens terapêuticas não cirúrgicas, que auxiliem no processo de regeneração e melhora funcional após a lesão nervosa, dentre as quais destaca-se o exercício físico [4]. Contudo, em muitos casos, a etiologia da lesão é mal compreendida, resultando em pouca eficiência dos cuidados prestados [5].

O exercício físico é um tratamento eficaz para vários transtornos dolorosos [6]. O exercício aeróbico ou resistido provoca um aumento generalizado da tolerância à dor, durante e imediatamente após o exercício, podendo esse efeito estender-se por até 30 minutos após o seu término. Os mecanismos fisiológicos que explicam a ação do exercício sobre a dor não estão completamente esclarecidos, mas dados de pesquisas sugerem que o exercício físico provoca a liberação de opióides endógenos e ativa mecanismos inibitórios nociceptivos na coluna vertebral, levando à diminuição da sensação dolorosa [7-9].

Há evidências na literatura de que o exercício físico atua também sobre o processo inflamatório, visto que a contração muscular estimula a liberação de citocinas anti-inflamatórias e inibe a produção de citocinas pró-inflamatórias, auxiliando na atenuação desse processo [10]. Também é relatado que os efeitos do exercício sobre o processo inflamatório estão diretamente relacionados com a intensidade do mesmo [11]. Além disso, o exercício físico pode provocar alterações sobre a morfologia do nervo periférico lesionado, influenciando a velocidade de regeneração axonal e promovendo maior expressão de proteínas que estimulam o crescimento neural [12].

Dessa forma, observa-se o quanto o exercício físico pode ter um papel importante na recuperação de lesões nervosas periféricas. Contudo, a modalidade de exercício resistido tem sido pouco estudada, em comparação com o exercício aeróbico [11]. Este estudo objetivou analisar os efeitos do exercício físico resistido, de subida em escada, sobre o edema, nocicepção e regeneração do nervo isquiático de ratos Wistar, submetidos a modelo experimental de ciatalgia.

\section{MÉTODOS}

O presente estudo caracteriza-se como sendo uma pesquisa experimental de natureza quantitativa. A pesquisa foi aprovada pelo Comitê de Ética no Uso de Animais da Universidade Estadual do Oeste do Paraná (Unioeste) em 04/2014, e conduzido segundo as normas internacionais de ética em experimentação animal [13].

\section{Grupos experimentais}

O grupo amostral foi composto por 24 ratos da linhagem Wistar [14], machos, com idade média de 10 semanas, pesando em média 352 gramas. Os animais ficaram alojados em caixas padrão de polipropileno, em ambiente com temperatura controlada, em torno de $23 \pm 1^{\circ} \mathrm{C}$, com fotoperíodo de 12 horas, recebendo água e ração ad libitum.

Os animais foram distribuidos aleatoriamente em quatro grupos, compostos por seis animais cada: GC - Grupo Controle: os animais não foram lesionados nem submetidos ao protocolo de exercício. GE Grupo Exercício: os animais não foram lesionados, mas realizaram o protocolo de exercício resistido de subida em escada. GL - Grupo Lesão: os animais foram somente lesionados. GLE - Grupo tratado (Lesão e Exercício): os animais foram lesionados e submetidos ao protocolo de tratamento com exercício resistido de subida em escada.

\section{Protocolo de produção da lesão}

Os animais dos grupos submetidos à lesão foram previamente pesados e anestesiados com cloridrato de quetamina $(95 \mathrm{mg} / \mathrm{kg})$ e cloridrato de xilazina $(12 \mathrm{mg} / \mathrm{kg})$ por via intraperitoneal. Após verificação do estado de consciência do animal, por meio do 
pinçamento da cauda e pregas interdigitais, foi realizada a tricotomia da coxa posterior direita e assepsia da região com uso de povidine. Em seguida, foi feita uma incisão, paralela às fibras do bíceps femoral, expondo o nervo isquiático, e subsequente compressão do mesmo, com pinça hemostática, durante 30 segundos [15], com a finalidade de reproduzir dor no trajeto do nervo. A pressão de pinçamento foi padronizada para todos os animais, utilizando como referência o segundo dente da cremalheira da pinça hemostática, sendo sempre realizada pelo mesmo pesquisador. Após o pinçamento foi realizada uma marcação no local da lesão, por sutura epineural, utilizando fio de nylon 10.0, a fim de facilitar a localização da região lesionada [16]. Por fim, foi feita a sutura por planos e aplicado povidine sobre o local da incisão. Em seguida, os animais foram alojados nas mesmas condições pré-cirúrgicas.

\section{Protocolo de tratamento com exercício resistido de subida em escada}

Para a realização do tratamento com exercício resistido foi utilizada uma escada vertical de madeira, que possuía 67 degraus de ferro, altura de $1,18 \mathrm{~m}$, largura de $20,5 \mathrm{~cm}$ e inclinação de $60^{\circ}$. No alto da escada, foi posicionada uma caixa escura, com altura de $18,5 \mathrm{~cm}$ e largura de $15 \mathrm{~cm}$, em que os animais descansavam entre uma série e outra de exercício [17].

O procedimento consistiu na realização pelo animal de duas séries de 10 subidas na escada, com sobrecarga de 100 gramas adaptada à sua cauda, com intervalo de um minuto entre uma série e outra. O protocolo começou a partir do terceiro dia após o procedimento cirúrgico $(\mathrm{PO})$ e foi conduzido por um período de três semanas, sendo realizadas 15 sessões de tratamento, com intervalo de dois dias a cada cinco sessões consecutivas de terapia. Para a realização desse tratamento, todos os animais foram previamente familiarizados com o equipamento durante duas semanas.

\section{Avaliação do edema}

Para o procedimento de avaliação do edema (AVE) foi utilizado o membro pélvico direito de todos os animais. Foi considerada a alteração volumétrica de líquidos (em ml), por meio de hidropletismografia, com a utilização de um pletismômetro de pata da marca Insight ${ }^{\circledR}$ (Ribeirão Preto, SP). Foi introduzido o membro pélvico direito dos animais, até o nível do tornozelo, em um recipiente com água, e pelo deslocamento da água o equipamento quantificava o edema. Os procedimentos de AVE iniciaram no $3^{\circ} \mathrm{PO}$, sendo algumas avaliações feitas antes do exercício e algumas após o exercício. AVE 1 ocorreu no $3^{\circ} \mathrm{PO}$, antes da primeira sessão de exercício; AVE 2 ocorreu nesse mesmo dia, logo após o exercício; AVE 3 no $7^{\circ} \mathrm{PO}$, após o exercício; AVE 4 no $10^{\circ} \mathrm{PO}$, antes do exercício; AVE 5 no $14^{\circ} \mathrm{PO}$, após o exercício; AVE 6 no $21^{\circ} \mathrm{PO}$, após o exercício; e a última avaliação, AVE 7, ocorreu no $22^{\circ} \mathrm{PO}$, antes da eutanásia dos animais.

\section{Avaliação da nocicepção}

Para avaliação da nocicepção (AVN) foi utilizado o limiar de retirada à pressão, com auxílio de um analgesímetro digital, tipo filamento de Von Frey, da marca Insight ${ }^{\circledR}$ (Ribeirão Preto, SP). O equipamento consiste de um braço com ponteira descartável de polipropileno, com a capacidade de avaliar entre 0,1-1000 g, ligado a uma caixa amplificadora, ou seja, é um transdutor de pressão adaptado a um contador digital de força expressa em gramas [18].

O filamento foi aplicado no local da lesão do animal. Dessa maneira, para realização do teste no local da lesão, o animal foi contido manualmente e a ponta do filamento foi aplicada perpendicularmente à área da incisão cirúrgica, exercendo-se uma força com aumento gradual, até que o animal retirasse seu membro, e então o teste era interrompido para registro do limiar de retirada. Para facilitar a adaptação dos animais a esse instrumento, nos três dias anteriores à lesão foi realizada a simulação desta avaliação [19].

As AVN ocorreram nos seguintes momentos: AVN 1 imediatamente anterior à lesão; AVN 2 no $3^{\circ}$ dia de PO anteriormente ao exercício; AVN 3 nesse mesmo dia, logo após o exercício; AVN 4 no $7^{\circ} \mathrm{PO}$ após o exercício; AVN 5 no $10^{\circ} \mathrm{PO}$ antes do exercício; AVN 6 no $14^{\circ}$ PO após o exercício; AVN 7 no $21^{\circ}$ PO após o exercício; e a última avaliação, AVN 8, ocorreu no $22^{\circ} \mathrm{PO}$, anteriormente à eutanásia dos animais.

\section{Eutanásia dos animais e coleta das amostras}

No $22^{\circ} \mathrm{PO}$, os animais foram pesados e anestesiados, sendo dissecado e coletado um fragmento de $2 \mathrm{~cm}$ do nervo isquiático posterior direito dos animais, distal ao procedimento de compressão. Após isso, ainda sob efeito do anestésico, os animais foram eutanasiados por meio de decapitação em guilhotina. 


\section{Análise morfométrica}

Os fragmentos dos nervos coletados foram fixados em paraformoldeído $4 \%$ e pós-fixados em tetróxido de ósmio; posteriormente foram processados em técnica histológica de rotina e submetidos a cortes transversais de 5 micrômetros $(\mu \mathrm{m})$ de espessura [20].

A partir das lâminas histológicas confeccionadas, foi utilizada a objetiva de 100X, para fotomicrografar no aparelho Olympus BX 50, (Olympus America Inc., NY, USA) imagens em quatro campos visuais, localizados sistematicamente em quadrantes superior esquerdo (Q1), superior direito (Q2), inferior direito (Q3) e inferior esquerdo (Q4) [21].

A partir desses quadrantes foi realizada a estimativa do número de axônios e da densidade de fibras nervosas, utilizando o programa Image Pro-Plus 6.0, sendo contabilizados todos os axônios de cada um dos quatro quadrantes e somado, considerando que foram incluídos na contagem os axônios que estivessem tocando as bordas denominadas "de inclusão" (superior e esquerda) e excluindo os que tocassem as bordas "de exclusão" (inferior e direita). Contudo para a densidade de fibras nervosas, o número de axônios foi dividido pela área dos quadrantes, a fim de se obter uma estimativa da quantidade de fibras nervosas por micrômetro $\left(\mu \mathrm{m}^{2}\right)$ de quadrante.

\section{Fator de Crescimento Neural e Fator de Crescimento Derivado do Cérebro}

Foram avaliadas as expressões de dois fatores de crescimento neural no nervo isquiático, o Fator de Crescimento Neural (Neural Growth Factor, NGF) e o Fator de Crescimento Derivado do Cérebro (Brain-derived Neurotrophic Factor, BDNF), por meio da técnica de Western blotting. Para tanto, um fragmento do nervo isquiático foi retirado e colocado em solução de RNA Later por 24 horas, sendo após homogeneizado em tampão de extração. Os extratos foram centrifugados a $15000 \mathrm{rpm} \mathrm{a} 4^{\circ} \mathrm{C}$ por 40 minutos, para remoção do material insolúvel. A concentração proteica foi determinada usando o método de Bradford com reagente da BioRad $\left(\right.$ BioRad $\left.^{\circledR}, \mathrm{CA}, \mathrm{USA}\right)$. As amostras foram tratadas com tampão Laemmli contendo Ditiotreitol (DTT) $10 \mathrm{mM}$, aquecidas em água fervente por $5 \mathrm{~min}$. Alíquotas com concentrações proteicas iguais foram aplicadas no sodium dodecyl sulfate-polyacrylamide gel electrophoresis (SDSPAGE) (6 a 10\% de Trisacrilamida) em aparelho minigel com marcadores de pesos moleculares conhecidos. Após corridas, as proteínas foram transferidas para membrana de nitrocelulose, a qual foi incubada por duas horas em solução bloqueadora para diminuir a ligação inespecífica das proteínas. A seguir as membranas foram incubadas com anticorpos específicos para as diferentes proteínas a serem analisadas. Subsequentemente, as membranas foram incubadas por uma hora com anticorpo secundário. As bandas proteicas específicas foram detectadas por quimioluminecência e expostas a filmes fotográficos. As intensidades das bandas foram quantificadas por densiometria óptica.

\section{Análise estatística}

A normalidade dos dados foi analisada por meio do teste de Shapiro-Wilk. Em seguida, os resultados da avaliação funcional foram submetidos à estatística inferencial pelo teste de ANOVA misto. Os resultados da análise morfométrica foram analisados pelo ANOVA unidirecional (apresentados pela estatística F, e os respectivos graus de liberdade e erro) e os resultados da análise molecular foram submetidos ao teste não paramétrico de Kruskal-Wallis, com pós-teste de Dunn. Em todos os casos o nível de significância aceito foi de $\alpha=5 \%$.

\section{RESULTADOS}

\section{Edema do membro pélvico direito dos animais}

Para os valores de edema, foi possível observar diferença significativa somente entre as avaliações $\mathrm{F}(6 ; 15,05)=13,03 ; \mathrm{p}<0,001$ - não ocorrendo diferença entre os grupos. Desta maneira, os resultados da AVE 7 foram menores do que todos os outros, sendo os seguintes valores de $\mathrm{p}$ : AVE 7 comparada a AVE 1 $(p=0,001)$, AVE 7 comparada a AVE 2 e AVE 3 $(p<0,001)$, AVE 7 comparada a AVE $4(p=0,020)$, AVE 7 comparada a AVE $5(\mathrm{p}=0,002)$ e AVE 7 comparada a AVE $6(p=0,018)$. Também AVE 3 mostrou resultados significativos e maiores quando comparados a todas as subsequentes avaliações, dessa maneira os seguintes valores de $\mathrm{p}$ foram obtidos: AVE 3 comparada a AVE 4, AVE 5 e AVE $7(p<0.001)$ e comparada a AVE $6(p=0,004)$ (Tabela 1).

\section{Nocicepção no local da lesão}

Observando os dados das oito avaliações realizadas, foi possível observar diferença significativa - $F(7 ; 14,76)=34,22 ; p<0,001$ - sendo que o GC apresentou valor significativamente maior que os demais $(p<0,001)$. Para as comparações entre as avaliações, foi possível observar que: AVN 1 foi 
menor comparada a AVN 7 e AVN $8(\mathrm{p}<0,001)$. AVN 2 também foi menor comparada com AVN 4 em diante, com o valor de $p$ oscilando entre $p<0,001$ a $\mathrm{p}=0,009$. AVN 3 foi menor comparada a AVN 5-AVN 8, com $\mathrm{p}<0,001$ a $\mathrm{p}=0,035$. AVN 4 foi menor comparada a AVN 6-AV 8, com $\mathrm{p}<0,001$ a $\mathrm{p}=0,013$. AVN 5 foi menor comparada às duas últimas, sendo $p=0,001$ e $p<0,001$ respectivamente. E AVN 6 foi menor comparada a AVN 8, tendo um $\mathrm{p}=0,001$ (Tabela 2).

\section{Número de axônios}

Não houve diferença significativa entre os grupos: $\mathrm{F}(3 ; 20)=0,5412 ; \mathrm{p}=0,6632$.

\section{Densidade de fibras nervosas}

Não houve diferença na densidade das fibras nervosas entre os grupos: $\mathrm{F}(3 ; 20)=0,5412 ; \mathrm{p}=0,6632$.

\section{Fator de Crescimento Neural e Fator de Crescimento Derivado do Cérebro}

Quanto à expressão de NGF, não foi possível observar diferença significativa entre os grupos $(\mathrm{p}=0,0563)$ (Figura 1A). Entretanto, na análise do BDNF, obtiveram-se diferenças significativas comparando o GC com GL e GLE $(\mathrm{p}<0,005)$, sendo que GC obteve valores menores (Figura 1B).

Tabela 1. Dados referentes à estimativa de edema da pata (inserção até articulação talocrural) no membro posterior direito dos animais submetidos ou não ao exercício resistido de subida em escada.

\begin{tabular}{lcccc}
\hline \multicolumn{1}{c}{ Avaliações } & \multicolumn{4}{c}{$\begin{array}{c}\text { Grupos } \\
\text { Mililitros (média } \pm \text { desvio padrão) }\end{array}$} \\
\cline { 2 - 5 } & $\begin{array}{c}\text { Grupo Controle } \\
\text { (GC) }\end{array}$ & $\begin{array}{c}\text { Grupo Exercício } \\
\text { (GE) }\end{array}$ & $\begin{array}{c}\text { Grupo Lesão } \\
\text { (GL) }\end{array}$ & $\begin{array}{c}\text { Grupo Tratado } \\
\text { (GLE) }\end{array}$ \\
\hline Avaliação 1 & $2,38 \pm 0,23$ & $1,99 \pm 0,31$ & $1,93 \pm 0,37$ & $2,07 \pm 0,35$ \\
Avaliação 2 & $2,34 \pm 0,49$ & $1,79 \pm 0,44$ & $2,13 \pm 0,33$ & $2,3 \pm 0,37$ \\
Avaliação 3* & $2,28 \pm 0,26$ & $2,38 \pm 0,28$ & $2,29 \pm 0,21$ & $1,83 \pm 0,31$ \\
Avaliação 4 & $2,09 \pm 0,24$ & $1,95 \pm 0,20$ & $1,96 \pm 0,39$ & $1,92 \pm 0,06$ \\
Avaliação 5 & $1,78 \pm 0,26$ & $2,15 \pm 0,19$ & $2,05 \pm 0,18$ & $2 \pm 0,31$ \\
Avaliação 6 & $1,90 \pm 0,46$ & $2,01 \pm 0,22$ & $1,94 \pm 0,22$ & $1,83 \pm 0,11$ \\
Avaliação 7* & $1,89 \pm 0,37$ & $1,59 \pm 0,28$ & $1,45 \pm 0,17$ & $2,04 \pm 0,25$ \\
\hline Média & $2,09 \pm 0,33$ & $1,950,27$ & $1,96 \pm 0,26$ & \\
\hline
\end{tabular}

(*) Diferença significativa comparada às demais avaliações, considerando $p<0,05$.

Tabela 2. Dados referentes a nocicepção no local da lesão, no membro posterior direito dos animais, submetidos ou não ao exercício resistido de subida em escada.

\begin{tabular}{|c|c|c|c|c|}
\hline \multirow{2}{*}{ Avaliações } & \multicolumn{4}{|c|}{$\begin{array}{c}\text { Grupos } \\
\text { Gramas (média } \pm \text { desvio padrão) }\end{array}$} \\
\hline & $\begin{array}{l}\text { Grupo Controle } \\
\text { (GC) }\end{array}$ & $\begin{array}{l}\text { Grupo Exercício } \\
\text { (GE) }\end{array}$ & $\begin{array}{l}\text { Grupo Lesão } \\
\text { (GL) }\end{array}$ & $\begin{array}{l}\text { Grupo Tratado } \\
\text { (GLE) }\end{array}$ \\
\hline Avaliação 1* & $296,27 \pm 44,35$ & $222,70 \pm 41,99$ & $185,57 \pm 37,15$ & $229,29 \pm 38,62$ \\
\hline Avaliação 2* & $227,33 \pm 23,71$ & $212,20 \pm 43,32$ & $217,19 \pm 28,82$ & $203,70 \pm 29,72$ \\
\hline Avaliação 3* & $287,16 \pm 43,22$ & $214,45 \pm 29,85$ & $200,57 \pm 28,27$ & $203,54 \pm 40,02$ \\
\hline Avaliação 4* & $284,44 \pm 28,90$ & $212,58 \pm 32,33$ & $242,19 \pm 34,86$ & $262,37 \pm 23,50$ \\
\hline Avaliação 5* & $354,38 \pm 56,39$ & $193,08 \pm 33,39$ & $262,00 \pm 28,64$ & $232,15 \pm 15,61$ \\
\hline Avaliação 6* & $365,83 \pm 78,73$ & $244,12 \pm 54,59$ & $245,38 \pm 43,24$ & $261,12 \pm 22,70$ \\
\hline Avaliação 7 & $327,94 \pm 0,37$ & $308,45 \pm 30,78$ & $323,19 \pm 32,17$ & $282,45 \pm 35,28$ \\
\hline Avaliação 8 & $338,77 \pm 31,05$ & $332,20 \pm 51,73$ & $347,95 \pm 28,93$ & $307,29 \pm 27,63$ \\
\hline Média & $310,26 \pm 38,34$ & $242,47 \pm 39,74^{\dagger}$ & $252,99 \pm 32,76^{+}$ & $247,73 \pm 29,13^{+}$ \\
\hline
\end{tabular}

* Diferenças significativas comparadas a avaliações subsequentes.

${ }^{\dagger}$ Diferença significativa comparada com GC, considerando $\mathrm{p}<0,05$. 


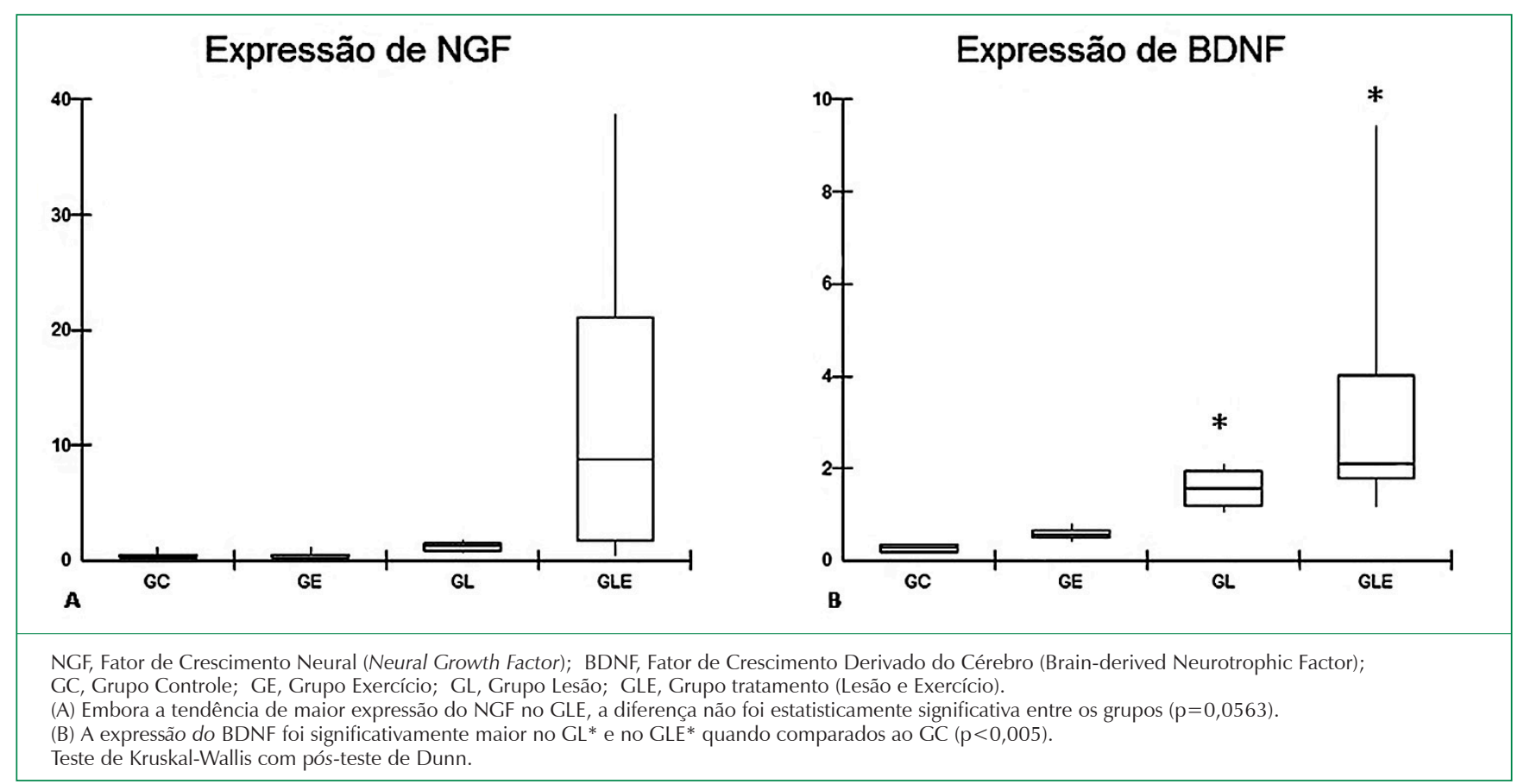

Figura 1. Gráficos representativos da expressão proteica.

\section{DISCUSSÃO}

Este estudo, conduzido por 22 dias, testou um programa de treinamento físico resistido de subida em escada, em ratos Wistar, visando verificar a influência desse exercício no edema, no limiar nociceptivo e na morfometria do nervo isquiático, após indução de lesão nervosa periférica.

Com relação à análise dos resultados sobre o edema, as primeiras avaliações apresentaram valores maiores que as últimas, o que pode indicar que nos primeiros dias pós-indução da lesão nervosa, os animais apresentavam mais edema, o que está de acordo com o que a literatura apresenta, visto que, segundo Grinsell e Keatin [22], o período entre 24 a 48 horas posteriores à lesão é aquele em que está ocorrendo maior acúmulo de macrófagos e células de Schwann no local da lesão, denotando um aumento do processo inflamatório local. Contudo, quando foram comparados os resultados de edema intragrupos, não foi possível observar diferença estatisticamente significativa.

Por outro lado, a redução nos valores de deslocamento de água, observada com o passar do estudo, pode indicar hipotrofia muscular, e não apenas redução de edema, ou seja, durante o período do estudo, a musculatura esquelética inervada pelo nervo isquiático pode ter sofrido hipotrofia, o que consequentemente redu- ziria os valores de deslocamento de água. Zhao et al. [23] demonstraram que a lesão do nervo isquiático causou atrofia e hipotrofia do músculo gastrocnêmio, em ratos da linhagem Sprague-Dawley, a partir de duas semanas após a lesão. Contudo, a literatura mostra que o exercício físico pode auxiliar na atenuação do processo inflamatório, pois a ativação de fibras musculares induz à produção de interleucina (IL)-6, que, no músculo, atua como uma citocina anti-inflamatória, estimulando o aparecimento na circulação de outras citocinas antiinflamatórias, tais como IL-1ra e IL-10, além de inibir a produção da citocina pró-inflamatória TNF- $\alpha$ [11].

Não foi encontrada diferença estatisticamente significativa na comparação entre os grupos, o que pode estar relacionado aos parâmetros do exercício aplicado. No estudo de English et al. [12], que teve como objetivo comparar os efeitos do exercício físico aeróbico de baixa e alta intensidade em esteira ergométrica sobre a resposta inflamatória aguda em ratos Wistar, foi utilizado um protocolo de exercício físico de baixa e alta intensidade. Os autores concluíram que o exercício de alta intensidade foi mais eficaz na redução do edema inflamatório [12]. Porém, há uma linha tênue para intensidade do exercício, pois se for extenuante pode provocar lesão muscular e ativar citocinas pró-inflamatórias, aumentando o processo inflamatório [11]. 
No estudo de Antunes et al. [24] foram verificados os efeitos do exercício físico resistido de salto em meio aquático sobre o edema inflamatório, causado por lesão traumática no tendão calcâneo dos animais. O protocolo de exercício consistiu em realizar duas séries de cinco saltos ou quatro séries de cinco saltos na água por uma semana. Com isso, constataram que o exercício foi ligeiramente benéfico na redução do edema, sendo o segundo protocolo mais eficaz. Cabe destacar também que, no presente estudo, não foi realizada uma avaliação de edema pré-lesão, o que impossibilitou uma comparação com valores pré cirúrgicos, sendo esta uma limitação. Ainda, o teste mais utilizado para avaliação funcional, que é o índice de funcionalidade do isquiático, não foi realizado devido a dificuldades técnicas, sendo uma importante limitação deste estudo.

Sobre os resultados da análise nociceptiva, observou-se que com o passar das avaliações o limiar nociceptivo aumentou dentro de cada grupo. Entretanto, na comparação entre os grupos, somente houve resultado estatisticamente significativo comparando o grupo controle com os demais, mostrando que a lesão foi efetiva para alterar o limiar nociceptivo, e o tratamento não foi eficiente para retornar os valores basais.

O uso do exercício físico é utilizado como uma abordagem não farmacológica para o tratamento da dor neuropática periférica. Esta foi a conclusão do estudo de Belotto et al. [25], que testou o efeito do exercício de natação na alodínia ao frio e hiperalgesia ao calor, num modelo de ciatalgia, em ratos Sprague-Dawley e camundongos CD1. Observaram que em 18 a 25 dias de natação após lesão nervosa diminuiu a alodínia ao frio e a hiperalgesia térmica nos animais, e já com sete dias de natação, diminuiu a hiperalgesia térmica [25].

O fato de não ter ocorrido diferença estatisticamente significativa entre os grupos pode estar relacionado com o protocolo de exercício aplicado, que pode ter sido leve, visto que foram realizadas somente duas séries de 10 subidas na escada e não foram controlados valores de repetição máxima. Logo, poderia ter sido necessária a aplicação de um protocolo de exercício de maior intensidade, com controle de cargas progressivas e até individualizadas, sendo esta outra limitação apresentada por este experimento.

Galdino et al. [26] investigaram a influência de diferentes protocolos de exercício resistido, por meio de um modelo de levantamento de peso, sobre o limiar nociceptivo de ratas Wistar. O protocolo de exercício consistia em 15 séries de 15 repetições do exercício ou três séries de 10 repetições, com carga de até $75 \%$ de uma repetição máxima, durante 12 semanas, sendo que um grupo de animais foi submetido ao protocolo de exercícios associado ao uso de naloxona. Os autores observaram que o primeiro protocolo foi $o$ mais eficiente, aumentando significativamente o limiar nociceptivo, e que o grupo que associou o exercício com o uso de naloxona não obteve melhora da dor [26].

Em um experimento prévio realizado no mesmo laboratório do presente estudo, avaliou-se a eficácia do exercício físico de natação durante 30 minutos, com sobrecarga de $10 \%$ do peso corporal, e salto, com sobrecarga de $50 \%$ do peso corporal, sendo quatro séries de cinco saltos, por cinco sessões, sobre o quadro álgico de ratos Wistar, submetidos a um modelo experimental de ciatalgia. O exercício não foi eficaz para reduzir o quadro álgico dos ratos submetidos à ciatalgia [27]. Assim, cabe ressaltar o papel da intensidade do exercício sobre o efeito de redução da dor, o que, possivelmente, foi o principal motivo de não se ter encontrado diferença significativa entre os grupos deste estudo.

Outro fator que pode ter influenciado nos resultados do presente estudo foi o tempo transcorrido entre a realização do exercício e as avaliações, pois, como observado também no estudo de Galdino et al. [26], o efeito do exercício sobre a nocicepção ocorre por um período curto após o exercício, aproximadamente 15 minutos.

No presente estudo foi verificado o número de axônios e a média de densidade de fibras nervosas, sendo que para ambos, não foram obtidas diferenças significativas. Foram selecionados e contados os axônios em cada um dos quatro quadrantes, localizados na periferia da imagem, porém, posteriormente observou-se nas lâminas histológicas que a periferia do nervo geralmente apresentava-se com as fibras mais íntegras do que o centro do mesmo. Ou seja, mesmo nos grupos que sofreram lesões, as fibras nervosas periféricas estavam mais íntegras do que as centrais, o que pode ter influenciado os resultados.

Bonetti et al. [28] avaliaram a influência do exercício resistido sobre o grau de regeneração do nervo isquiático em ratos Wistar, pós lesão por pinçamento, baseando-se em analises morfométricas da porção distal do nervo. Uma das medidas realizadas nos nervos foi a densidade de fibras nervosas e, diferentemente do presente estudo, aqueles autores constataram que o grupo exercitado obteve valores menores de densidade quando comparado ao grupo placebo e grupo lesão. Os autores sugeriram que isso pode ter ocorrido porque o grupo lesão apresentava grande quantidade de fibras, embora com diâmetros menores. 
Quanto à expressão de NGF e BDNF, observou-se que BDNF apresentou elevação nos grupos em que houve lesão, fato concordante com a literatura [22]. Entretanto, o grupo que recebeu exercício não foi diferente daquele apenas lesado, o que também ocorreu para o grupo sem lesão, mas com exerício. Berchtold et al. [29] submeteram ratos Sprague-Dawley a exercício em esteira por quatro semanas e avaliaram a expressão do BDNF no sistema nervoso central dos animais, verificando que o exercício aumentou os níveis de BDNF no hipocampo.

Conclui-se que o exercício físico resistido de subida em escada, nos parâmetros utilizados, não foi eficaz para reduzir o edema, aumentar o limiar nociceptivo, a quantidade e densidade de fibras nervosas, ou alterar significativamente a expressão de NGF e BDNF, neste modelo experimental de ciatalgia.

\section{NOTAS}

Apoio financeiro

Os autores agradecem à Coordenação de Aperfeiçoamento de Pessoal de Nível Superior (CAPES), pela concessão da bolsa de mestrado para J. S. A.

Declaração de conflitos de interesse

Os autores declaram não haver conflitos de interesse relevantes ao conteúdo deste estudo.

\section{REFERÊNCIAS}

1. Hoy D, Bain C, Williams G, March L, Brooks P, Blyth F, Woolf A, Vos T, Buchbinder R. A systematic review of the global prevalence of low back pain. Arthritis Rheum. 2012;64(6):2028-37. http://dx.doi.org/10.1002/art.34347

2. Koes BW, Van Tulder MW, Thomas S. Diagnosis and treatment of low back pain. BMJ. 2006 June 17;332(7555):1430-4. http://dx.doi. org/10.1136/bmj.332.7555.1430

3. Stafford MA, Peng P, Hill DA. Sciatica: a review of history, epidemiology, pathogenesis, and the role of epidural steroid injection in management. Br J Anaesth. 2007;99(4):461-73. http://dx.doi.org/10.1093/bja/aem238

4. Albornoz PM, Delgado PJ, Forriol F, Maffulli N. Non-surgical therapies for peripheral nerve injury. Br Med Bull. 2011;100(73):1-28. http://dx.doi.org/10.1093/bmb/ldr005

5. Dreisinger TE. Exercise in the management of chronic back pain. Ochsner J. 2014;14(1):101-7.

6. Oliveira MAS, Fernandes RSC, Daher SS. Impacto do exercício na dor crônica. Rev Bras Med Esporte. 2014; 20(3):200-3. http://dx.doi. org/10.1590/1517-86922014200301415

7. Nijs J, Kosek E, Oosterwijck JV, Meeus M. Dysfunctional endogenous analgesia during exercise in patients with chronic pain: to exercise or not to exercise? Pain Physician. 2012;15(3 Suppl):ES205-13.

8. Souza JB. Poderia a atividade fisica induzir analgesia em pacientes com dor cronica? Rev Bras Med Esporte. 2009;15(2):145-50. http:// dx.doi.org/10.1590/S1517-86922009000200013

9. Mazzardo-Martins L, Martins DF, Marcon R, Santos UD, Speckhann B, Gadotti V, Siqwalt AR, Guglielmo LG, Santos AR. High-intensity extended swimming exercise reduces pain-related behavior in mice: involvement of endogenous opioids and the serotonergic system. J Pain. 2010;11(12):1384-93. http://dx.doi.org/10.1016/j.jpain.2010.03.015

10. Petersen AMW, Pedersen BK. The anti-inflammatory effect of exercise. J Appl Physiol. 2005;98(1)1154-62. http://dx.doi.org/10.1152/ japplphysiol.00164.2004

11. Lana AC, Paulino CA, Goncalves ID. Efeitos dos exercícios físicos sobre o edema inflamatório agudo em ratos Wistar. Rev Bras Med Esporte. 2008;14(1)33-7. http://dx.doi.org/10.1590/S1517-86922008000100006

12. English AW, Wilhelm JC, Sabatier MJ. Enhancing recovery from peripheral nerve injury using treadmill training. Ann Anat. 2011;193(1)35461. http://dx.doi.org/10.1016/j.aanat.2011.02.013

13. Schnaider TB, Souza C. Aspectos éticos da experimentação animal. Rev Bras Anestesiol. 2003;53(2)278-85. http://dx.doi.org/10.1590/ S0034-70942003000200014

14. Gaffuri J, Meireles A, Rocha BP, Rosa CT, Artifon EL, Silva LI, Moreira NB, Bertolini GRF. Avaliação do exercício físico como fator de analgesia em um modelo experimental de ciatalgia. Rev Bras Med Esporte. 2011;17(2)115-8. http://dx.doi.org/10.1590/S151786922011000200009

15. Bridge PM, Ball DJ, Mackinnon SE, Nakao Y, Brandt K, Hunter DA, Hertl C. Nerve crush injuries - a model for axonotmesis. Exp Neurol Suppl. 1994;127(2)284-90. http://dx.doi.org/10.1006/exnr.1994.1104

16. Mazzer PYCN, Barbieri CH, Mazzer N, Fazan VPS. Morphologic and morphometric evaluation of experimental acute crush injuries of the sciatic nerve of rats. J Neurosci Methods. 2008;173(2)249-58. http://dx.doi.org/10.1016/j.jneumeth.2008.06.019

17. Hornberger TA, Farrar RP. Physiological hypertrophy of the FHL muscle following 8 weeks of progressive resistance exercise in the rat. Can J Appl Physiol. 2004;29(1)16-31. http://dx.doi.org/10.1139/h04-002 
18. Vivancos GG, Verri Jr WA, Cunha TM, Schivo IRS, Parada CA, Cunha FQ, Ferreira SH. An electronic pressure-meter nociception paw test for rats. Braz J Med Biol Res. 2004 Mar;37(3):391-9. http://dx.doi.org/10.1590/S0100-879X2004000300017

19. Karvat J, Antunes JS, Bernardino GS, Kakihata CMM, Bertolini GRF. Effect of low-level laser and neural mobilization on nociceptive threshold in experimental sciatica. Rev Dor. 2014;15(3)207-10. http://dx.doi.org/10.5935/1806-0013.20140045

20. Di Scipio F, Raimondo S, Tos P, Geuna S. A simple protocol for paraffin-embedded myelin sheath staining with osmium tetroxide for light microscope observation. Microsc Res Tech. 2008;71(1)497-02. http://dx.doi.org/10.1002/jemt.20577

21. Geuna S, Tos P, Guglielmone R, Battiston B, Giacobini-Robecchi MG. Methodological issues in size estimation of myelinated nerve fibers in peripheral nerves. Anat Embryol. 2001;204(1)1-10. http://dx.doi.org/10.1007/s004290100188

22. Grinsell D, Keating CP. Peripheral nerve reconstruction after injury: a review of clinical and experimental therapies. Biomed Res Int 2014;2014:698256. http://dx.doi.org/10.1155/2014/698256

23. Zao L, Guangming LV, Jiang S, Yan Z, Sun J, Wang L, Jiang D. Morphological differences in skeletal muscle atrophy of rats with motor nerve and/or sensory nerve injury. Neural Regen Res. 2012;7(32)2507-15. http://dx.doi.org/10.3969/j.issn.1673-5374.2012.32.004

24. Antunes JS, Karvat J, Meireles A, Rocha BP, Rosa CT, Silva LI, Bertolini GRF. Exercício resistido em meio aquático para ratos Wistar submetidos a trauma em tendão: avaliação da nocicepção e edema. Rev Dor. 2012;13(1)55-8. http://dx.doi.org/10.1590/S180600132012000100010

25. Belotto MF, Magdalon J, Rodrigues HG, Vinolo MAR, Curi R, Pithon-Curi TC, Hatanaka E. Moderate exercise improves leucocyte function and decreases inflammation in diabetes. Clin Exp Immunol. 2010;162(1)237-43. http://dx.doi.org/10.1111/j.1365-2249.2010.04240.x

26. Galdino GS, Duarte IDG, Perez AC. Participation of endogenous opioids in the antinociception induced by resistance exercise in rats. Braz J Med Biol Res. 2010;43(9)906-9. http://dx.doi.org/10.1590/S0100-879X2010007500086

27. Gaffuri J, Meireles A, Rocha BP, Rosa CT, Artifon EL, Silva LI, Moreira NB, Bertolini GRF. Avaliação do Exercício Físico Como Fator de Analgesia em Um Modelo Experimental de Ciatalgia. Rev Bras Med Esporte. 2011;17(2)115-8. http://dx.doi.org/10.1590/S151786922011000200009

28. Bonetti LV, Schneider APK, Barbosa S, Ilha L, Faccioni-heuser MC. Balance and coordination training and endurance training after nerve injury. Muscle Nerve. 2015;51(1)83-91. http://dx.doi.org/10.1002/mus.24268

29. Berchtold NC, Chinn G, Chou M, Kesslak JP, Cotman CW. Exercise primes a molecular memory for brain-derived neurotrophic factor protein induction in the rat. Neuroscience. 2005;133(3):853-61. http://dx.doi.org/10.1016/j.neuroscience.2005.03.026 